\section{Photochemistry and Absorption Spectrum of Acetone}

IT has been generally assumed that the explanation of the diffuse absorption spectra of aldehydes and ketones in the ultra-violet is the occurrence of a process of predissociation involving the splitting of a $\mathrm{C}-\mathrm{H}$ or $\mathrm{C}-\mathrm{C} \operatorname{link}^{1}$. Against this observation are the following observations :

(1) The vapours of aldehydes and ketones exhibit fluorescence.

(2) The unimolecular decomposition is always accompanied by bimolecular polymerisation.

(3) The quantum efficiency of decomposition is diminished on passing from $\mathrm{C}-\mathrm{H}$ to $\mathrm{C}-\mathrm{C}$ compounds.

(4) Complex ketones decompose in quite a different way from acetone, giving very little carbon monoxide.

(5) The photodecomposition is not a chain reaction.

The photo-reactions of acetone illustrate (1) and (2). In the gaseous state it decomposes with a quantum efficiency of about $\mathbf{0 . 2}$ only $^{2}$, and we have found that in the liquid state it polymerises (without decomposition) with about the same quantum efficiency. As it is difficult to assume a back reaction to explain the low efficiency ${ }^{3}$, it seems more probable that no splitting of a link occurs in the excited molecule. Instead, two processes may occur : (i) bimolecular interaction to give polymerisation, (ii) unimolecular decomposition through the similar interaction of two parts of the molecule, for example,

$$
\underset{\mathrm{CH}_{3}}{\mathrm{CH}_{3}}>\mathrm{CO} \rightarrow \underset{\mathrm{CH}_{3}}{\mathrm{CH}_{3}}+\mathrm{CO} .
$$

In the case of the ketone $\mathrm{CH}_{3} \mathrm{CH}_{2} \mathrm{CH}_{2} \mathrm{CH}_{2}>\mathrm{CO}$ the products are not unexpectedly $\mathrm{CH}_{3}-\underset{\mathrm{CH}}{\mathrm{CH}}$ and $\mathrm{CH}_{3}>\mathrm{CO}^{4}$

Unless the above unimolecular dissociation takes place within a rotational period it becomes necessary to find another explanation of the diffuseness of the absorption spectrum of some of these substances. We have recently examined the absorption spectrum of acetone, using pressures $0.5-200 \mathrm{~mm}$. in absorbing columns up to one metre. With pressures higher than a few mm. a region of continuous absorption extends from $c .3200 \mathrm{~A}$. to $2400 \mathrm{~A}$., with a maximum at about $2800 \mathrm{~A}$. This is the region characteristic of compounds containing the $>\mathrm{C}=\mathrm{O}$ group. At lower pressures in longer columns and under higher dispersion (Hilger $E_{1}$ spectrograph) this continuum splits up into about four groups each containing about 25 diffuse bands. The centres of the respective groups lie at c. 3150, 2900, 2710 and 2570 A. The corresponding intervals are 2740,2420 and $2010 \mathrm{~cm}^{-1}$. (A strong Raman frequency of acetone is $2900 \mathrm{~cm}^{-1}$.) The width of the bands is of the order $2.5 \mathrm{~A}$. (c. $30 \mathrm{~cm}^{-1}$ ) and their separation uniformly about $4 \mathrm{~A}$. With increasing pressure the bands widen and the groups extend so as to produce an effectively continuous absorption.

This type of equally spaced diffuse narrow diffuse bands is similar to that found in other $Y$-shaped molecules ${ }^{5}$. Assuming that the $\mathrm{CH}_{3}$ groups of acetone behave as single masses of 15 , and using probable interatomic distances, the moments of inertia of the
$Y$-shaped molecule are such that the rotation lines in the bands should be separated by only $c .0 .4 \mathrm{~cm} .^{-1}$. There will, moreover, be a double series of $P$ and $R$ branches. It seems therefore inherently impossible to detect the fine structure in this spectrum, and the diffuseness of the bands can be attributed to an unresolved close packing of the rotation lines without calling upon the additional hypothesis of predissociation.

In the case of formaldehyde, where the moments of inertia are much smaller, it is not surprising that a region of fine structure is observed followed by diffuse bands, indicating unimolecular rearrangements within periods greater or less than those of rotation. E. J. BOWEN.

H. W. Thompson.

University College and

St. John's College,

Oxford.

Feb. 27.

${ }^{1}$ G. Herzberg, Trans. Far. Soc., 27, 378; 1931: R. Mecke, ibid., 359. 2 G. H. Damon and F. Daniels, J.A.C.S., 55, $2363 ; 1933$.

c.f. ${ }^{3} \mathrm{H}, \mathrm{O}$. Wig and G. B. Kistiakowsky, $J, C, S, 54,1806$ 1932 : R. O. Ogg, P. H. Leighton and F. W. Bergstrom, ibid., 56, 318 ; 1934.

4 R. G. W. Norrish, Trans, Far. Soc., 80, 103 ; 1934

V. Henri and O. R. Howell, Proc. Roy. Soc., A, 128, $192 ; 1930$.

\section{Activated Adsorption and Para-Ortho Hydrogen Conversion on Charcoal}

THe para-ortho hydrogen conversion was used by us among other reactions, at the suggestion of Prof. A. Frumkin, in order to investigate the chemical properties of hydrogen adsorbed on charcoal at high temperatures.

It has been shown in a qualitative way by Harkness and Emmett ${ }^{1}$ and by Rummel ${ }^{2}$ that adsorption of hydrogen on the surface of catalysts diminishes their activity in the ortho-para hydrogen conversion at $90^{\circ} \mathrm{K}$.

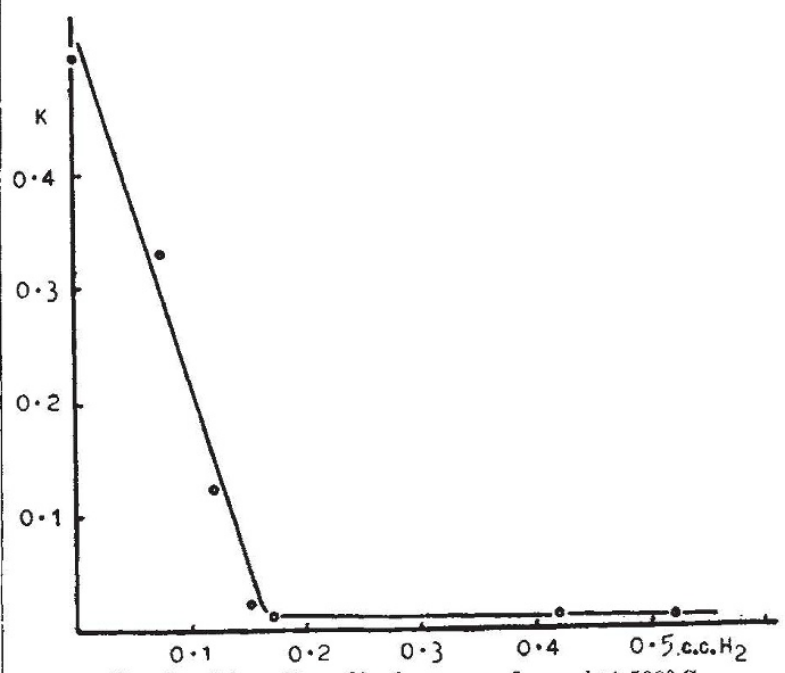

FIG. 1. Adsorption of hydrogen on charcoal at $500^{\circ} \mathrm{C}$.

We have investigated the relation between the velocity of the para-ortho conversion at $20^{\circ} \mathrm{C}$. and the quantity of gas adsorbed in the activated form. The charcoal was outgassed at $950^{\circ}$, then allowed to cool to the temperature of hydrogen adsorption, and after a definite amount of gas was adsorbed, further cooled to room temperature. The velocity of the 